\title{
OFF-SHELL SUPERSINGLETONS
}

\author{
E. Sokatchev ${ }^{*}$ ) \\ CERN - Geneva
}

\begin{abstract}
$\mathrm{N}=1,2,4$ supersingleton theories are formulated off-shell in superspace. It is shown that both the cases of $N=1$ and $N=2$ admit polynomial self-interactions, whereas non-polynomial ones exist in the case $\mathrm{N}=4$.
\end{abstract}

*) On leave of absence from the Institute for Nuclear Research and Nuclear Energy, Sofia, Bulgaria.

CERN-TH. $5033 / 88$

May 1988 


\section{Introduction}

Supersymmetric singletons are very special representations of the anti-de sitter supergroup OSp(N/4) [1-6]. Their peculiarity is that the supermultiplets are ultra-short, involving just a scalar and a spinor, both being spinors of the internal symmetry group $S O(N)$. An extensive study of singletons in view of their possible role as "preons" is being carried out by Flato, Fronsdal et al [2-6]. Recently the interest in singletons has broadened since they occur as solutions to the supermembrane equations upon compactification to $\operatorname{AdS}^{4} \mathrm{XS}^{\mathrm{N}-1}$ [7] . In this context an important question is whether there exist supersingleton interactions (it is related to the question if the supermembrane theory is a free one or not). In two recent papers $[8,9]$ it has been argued that such interactions exist only in the case $N=1$. In the present paper we develop an offshell description of supersingletons in superspace for the cases $N=1,2,4$ (a possibility of extending this result to $N=6$ is also suggested). We show that not only $N=1$, but also $N=2$ singletons admit polynomial self-interactions, whereas, in the case $\mathrm{N}=4$, non-polynomial ones (breaking so(4)) are still possible.

There are various ways of approaching an off-shell description of supersingletons. One option is to develop a superfield formalism in an anti-de sitter superspace having SO $(3,2) / \operatorname{SO}(3,1)$ as its even part (see $[10]$ for the case $N=1$ ). Another one is to realize the AdS group $S O(3,2)$ on the fivedimensional cone (see [4] where the first $N=1$ off-shell self- 
interacting singleton theory has been constructed). A third way, which we choose in the present paper, has been proposed in [8]. It consists of treating the supersingleton theory as a threedimensional Poincaré supersymmetric theory put in a specific conformal supergravity background. The latter is obtained by throwing away all the members of the supergravity multiplet except the dreibein, which is taken to describe the space $S^{2} \times S^{1}$ (the boundary of $\mathrm{AdS}^{4}$ at spatial infinity). Such an interpretation is possible because of the dual role played by the group SO $(3,2)$ : it is both the anti-de sitter group in four dimensions and the conformal group in three dimensions. We prefer this approach because it greatly simplifies the task of constructing field theories by dividing it into two parts. At first one considers supersymmetric field theories in flat threedimensional space, which is much easier than working directly in the curved anti-de sitter space. At the end one introduces the conformal supergravity background which is also not difficult, given the very restricted structure of the supergravity multiplet.

The above interpretation of the supersingleton theory gives a simple explanation of the fact that the singleton multiplets are so short. It is due to two reasons. First, in three dimensions a scalar $\phi$ and an on-shell spinor $\psi_{\alpha}$ field describe one degree of freedom each. Second, attaching spinor indices of So(N) to both $\phi$ and $\psi_{\alpha}$ and at the same time a vector index of SO(N) to the supersymmetry charges allows one to keep the onshell balance of bosons and fermions even in the extended case. In this way it is not hard to construct the free on-shell 
extended supersingleton theory. For example, in the case $N=8$ one finds $[8,9]$ :

$$
S=-1 / 2 \int d^{3} x \sqrt{-g}\left[g^{m n} \partial_{m} \phi^{a} \partial_{n} \phi^{a}+1 / 4 \kappa^{2} \phi^{a} \phi^{a}+i \bar{\psi}^{a} \gamma^{m} \nabla_{m} \psi^{\dot{a}}\right]
$$

Here $\phi^{a}$ and $\psi_{\alpha}^{\dot{a}}$ belong to the two inequivalent spinor representations of $\mathrm{SO}(\mathrm{N}), \nabla$ is the $\mathrm{S}^{2} \mathrm{XS} \mathrm{S}^{1}$ covariant derivative and $\kappa$ is the inverse radius of $S^{2} X S^{1}$. The action (1.1) is invariant under the following supersymmetry transformations:

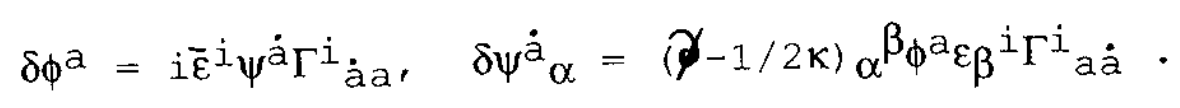

Their algebra closes on osp(8/4) only with the help of the equations of motion. The effects of introducing a specific conformal supergravity background in (1.1) are, first, the presence of the mass-like term $1 / 4 \kappa^{2} \phi^{a} \phi^{a}$ (it is actually the usual curvature term needed for weyl invariance [8]), and second, the killing spinor condition on the supersymmetry parameters $\varepsilon^{i}$. The latter originates from the choice $\psi_{\alpha}^{m i}=0$ for the gravitino field and the fact that it transforms inhomogeneously under local supersymmetry $\left(\delta \psi_{\alpha}^{m i} \sim \partial^{m} \varepsilon_{\alpha}{ }^{i}\right)$. Only certain backgrounds allow for such killing spinors, and $\mathrm{s}^{2} \mathrm{XS}{ }^{1}$ is one of them.

In order to study the possible self-interactions of supersingletons it is highly desirable to have an off-shell formulation of the theory. Finding it is, as usual, a nontrivial task which is greatly facilitated by employing superspace technique. Here we shall develop the formalism for 
the cases $\mathrm{N}=1,2,4$. Each case requires its own specific superspace. It will turn out that the $\mathrm{N}=2$ and $\mathrm{N}=4$ theories very much resemble the four-dimensional theories of the $N=1$ and $\mathrm{N}=2$ scalar multiplets. This is not surprising, since one way of obtaining extended supersymmetric theories in three dimensions is by dimensional reduction from four dimensions. Further, knowledge of how to treat conformal supergravity in four-dimensional superspace will help us a lot when introducing the $\mathrm{S}^{2} \mathrm{XS} \mathrm{s}^{1}$ background.

\section{2. $\mathbf{N}=1$ supersingletons}

The case $N=1$ is very simple indeed. It is well known how to formulate $N=1$ matter and supergravity theories in three dimensions (see, e.g., [11]). Here we shall consider this case mainly for pedagogical reasons.

The $\mathrm{N}=1$ singleton supermultiplet can be described by a three-dimensional scalar superfield

$\Phi(x, \theta)=\phi(x)+\theta^{\alpha} \psi_{\alpha}(x)+\theta^{2} F(x)$

Here $\theta^{\alpha}$ are the two component Majorana spinor Grassmann coordinates of superspace, and $F(x)$ is an auxiliary field. The action for $\Phi$ is

$S=\int d^{3} x d^{2} \theta\left[1 / 2 \Phi D^{\alpha}{ }_{D} \Phi+\lambda \Phi^{4}\right]$ 
where $D_{\alpha}=\partial_{\alpha}+i \theta^{\beta}\left(\gamma^{\mu}\right)_{\beta \alpha} \partial_{\mu}$

is the supersymmetric spinor derivative. The second term in (2.2) is a self-interaction with a dimensionless coupling constant $\lambda\left([\mathrm{dx}]=-1,[\mathrm{~d} \theta]=1 / 2,\left[D_{\alpha}\right]=1 / 2\right.$, so $[\Phi]=1 / 2$ and $[\lambda]=0)$. It is a simple exercise to show that the component field form of $(2.2)$ is

$s=\int d^{3} x\left[1 / 2 \phi 0 \phi+i / 2 \psi^{\alpha} \partial_{\alpha \beta} \psi^{\beta}-2 F^{2}+4 \lambda F \phi^{3}-3 \lambda \phi^{2} \psi^{\alpha} \psi_{\alpha}\right]$

putting (2.2) into an arbitraxy conformal supergravity background is also rather simple:

$S=\int d^{3} x d^{2} \theta E^{-1}\left[1 / 2 \Phi\left(D \alpha D_{\alpha}-R\right) \Phi+\lambda \Phi^{4}\right]$

Here $D_{\alpha}=E_{\alpha}{ }^{M} \partial_{M}+\omega_{\alpha}$ is the spinor derivative covariant with respect to the general coordinate transformation group $\delta \mathrm{x}^{\mathrm{m}}=$ $\lambda^{\mathrm{m}}(\mathrm{x}, \theta), \delta \theta_{\alpha}=\lambda^{\alpha}(\mathrm{x}, \theta)$ and involving supervielbeins $\mathrm{E}_{\alpha}{ }^{\mathrm{M}}(\mathrm{x}, \theta)$ and connection $\omega_{\alpha}(x, \theta)$. The term with the scalar curvature superfield $R(x, \theta)$ is needed for conformal invariance (just like in the $x$-space action). In our case $E_{\alpha}{ }^{M}$ and $\omega_{\alpha}$ correspond to the specialized background:

$$
\begin{aligned}
& E_{\alpha}{ }^{\mu}=\delta_{\alpha} \mu, E_{\alpha}{ }^{m}=i \theta^{\beta} e_{\alpha \beta^{m}}(x), \omega_{\alpha \beta} \beta^{\gamma}=\theta^{\rho} \omega_{\rho \alpha \beta} \beta^{\gamma(x)}, \\
& \left.E_{\alpha} \beta^{m}=e_{\alpha \beta^{m}}+\theta^{2}\left[e_{(\alpha}{ }^{\rho n} \partial_{n} e_{\beta}\right) \rho^{m}+\omega_{\alpha \beta^{\gamma}} \delta_{e_{\gamma} \delta^{m}}\right] .
\end{aligned}
$$

Here $e_{\alpha} \beta^{m(x)}$ and $\omega_{\alpha \beta} \beta^{\gamma \delta_{(x)}}$ are the dreibein and connection for $\mathrm{s}^{2} \mathrm{XS}{ }^{1}$. Putting all this into (2.5) quickly leads to the off- 
shell self-interacting $N=1$ singleton theory presented in $[8,9]$ and first derived as a five-dimensional cone theory in [4].

\section{3. $\mathbf{N}=2$ supersingletons}

In the case $N=2$ the internal symmetry is $S O(2) \sim U(1)$, so the Grassmann coordinates of superspace will carry a vector index, $\theta_{\alpha}{ }^{i}(i=1,2)$. In $U(1)$ notation we can represent $\theta_{\alpha}^{i}$ as $\left(\theta_{\alpha}{ }^{++}, \theta_{\alpha}{ }^{--}\right)$(the double U(1) charge ++ or -- distinguishes the projections of a vector from a spinor, which will carry single charge + or -). In ordinary real superspace,

$\left\{\mathrm{x}^{\mathrm{m}}, \theta_{\alpha}^{++}, \theta_{\alpha}^{--}\right\}$

supersymmetry is realized as follows:

$\delta x_{x}^{m}=i\left(\varepsilon^{--} \gamma^{m} \theta^{++}-\varepsilon^{++} \gamma^{m} \theta^{--}\right), \quad \delta \theta_{\alpha}^{ \pm \pm}=\varepsilon_{\alpha}^{ \pm \pm}$.

Making the following change of variables,

$z^{m}=x^{m}+i \theta^{--} \gamma^{m} \theta^{++}$,

one finds that the subspace

$\left\{z^{m}, \theta_{\alpha}^{++}\right\}$

is invariant under supersymmetry: 
$\delta z^{m}=2 i \varepsilon^{--} \gamma^{m} \theta^{++}, \quad \delta \theta_{\alpha}^{++}=\varepsilon_{\alpha}{ }^{++}$.

Similarly, one can consider the conjugate superspace $\left\{z^{m}, \theta_{\alpha}^{--}\right\}$. clearly, those superspaces are direct analogues of the fourdimensional $\mathrm{N}=1$ chiral superspace [12] (with left- or righthanded chirality replaced by $U(1)$ charges). A superfield defined in the superspace (3.4) and carrying an external charge + (i.e., a spinor index) has the following decomposition:

$\Phi^{+}\left(z, \theta^{++}\right)=\phi^{+}(z)+\theta^{\alpha++} \psi_{\alpha}^{-}(z)+\left(\theta^{++}\right) 2_{F}(-3)(z)$

The so(2) spinors $\phi^{+}$and $\psi_{\alpha}{ }^{-}$(together with their conjugates) are suitable for describing the $\mathrm{N}=2$ singleton multiplet, whereas $\mathrm{F}^{(-3)}$ is going to be auxiliary. Again in complete analogy with the four-dimensional $N=1$ case, the flat superspace action for $\Phi^{+}$and its conjugate $\Phi^{-}$is

$$
\begin{aligned}
S= & \int d^{3} x d^{2} \theta^{++} d^{2} \theta^{--} \Phi^{+}\left(x+i \theta^{--} \theta^{++}, \theta^{++}\right) \Phi^{-}\left(x-i \theta^{--} \theta^{++}, \theta^{--}\right)+ \\
& +\lambda \int d^{3} z d^{2} \theta^{++}\left(\Phi^{+}\right)^{4}+\lambda \int d^{3} z d^{2} \theta^{--}\left(\Phi^{-}\right)^{4} .
\end{aligned}
$$

Note that the self-interaction term is uniquely fixed if one insists on preserving SO(2) (the charge of the integrand $\left(\Phi^{+}\right)^{4}$ matches that of the measure $\mathrm{d}^{3} \mathrm{zd}^{2} \theta^{++}$). At the same time, from the kinetic term one concludes $[\Phi]=1 / 2$, so $[\lambda]=0$ and the self-coupling is conformally invariant. The evaluation of the component form of (3.7) is straightforward and we will not do it here (note that the derivative terms originate from the $\mathrm{x}$ shifts). 
The second part of our program is the introduction of the conformal supergravity background. Once again, we adapt the four-dimensional $\mathrm{N}=1$ formulation $[13,14,11]$. The gauge group of conformal supergravity is

$\delta z^{m}=\lambda^{m}\left(z, \theta^{++}\right), \quad \delta \theta^{\alpha++}=\lambda^{\alpha++}\left(z, \theta^{++}\right)$

with arbitrary parameters $\lambda$. The physical coordinate $x^{m}$ is defined as the real part of $\mathrm{z}^{\mathrm{m}}$ :

$x^{m}=1 / 2\left(z^{m}+\bar{z}^{m}\right)$,

whereas the imaginary part

$H^{m}\left(x, \theta^{++}, \theta^{--}\right)=-i / 2\left(z^{m}-\bar{z}^{m}\right)$

is the prepotential containing all the fields of conformal supergravity. Then the covariant form of (3.7) is

$S=\int d^{3} x d^{2} \theta^{++} d^{2} \theta^{--} E^{-1} \Phi^{+}\left(x+i H, \theta^{++}\right) \Phi^{-}\left(x-i H, \theta^{--}\right)+$

$$
+\lambda \int d^{3} z d^{2} \theta^{++}\left(\Phi^{+}\right)^{4}+\lambda \int d^{3} z d^{2} \theta^{--}\left(\Phi^{-}\right)^{4}
$$

Here $\boldsymbol{\Phi}^{+}$(and similarly for $\boldsymbol{\Phi}^{-}$) is assumed to transform under the group (3.8) as follows

$\boldsymbol{\Phi}^{+\prime}=\omega^{-1 / 4} \Phi^{+}, \quad\left(d^{3} z d^{2} \theta^{++}\right)^{\prime}=\omega\left(d^{3} z d^{2} \theta^{++}\right)$ 
The superdeterminant of vielbeins $E^{-1}$ has the transformation law

$E^{-1,}=E^{-1} \Omega^{-1}(\omega \bar{\omega})^{1 / 4}$,

$\left(d^{3} x d^{2} \theta^{++} d^{2} \theta^{--}\right)^{\prime}=\Omega\left(d^{3} x d^{2} \theta^{++} d^{2} \theta^{--}\right)$

The explicit expression for $\mathrm{E}^{-1}$ in terms of the prepotential $\mathrm{H}^{\mathrm{m}}$ is (see [13])

$E^{-1}=\left(\operatorname{det} q_{a}{ }^{m}\right)^{-1 / 2}\left[\operatorname{det}\left(1+H^{2}\right)\right]^{3 / 8}$

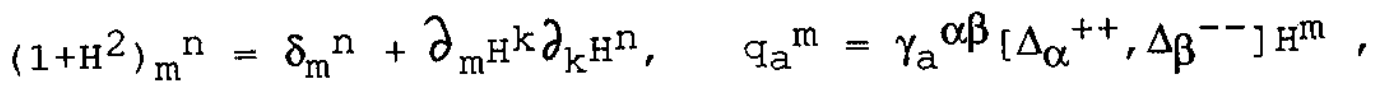

$\Delta_{\alpha}^{ \pm \pm}= \pm \partial_{\alpha}^{ \pm \pm}+i \partial_{\alpha}^{ \pm \pm} H^{m}\left(1 \mp i \partial_{H}\right)^{-1}{ }_{m}^{n} \partial_{n}$

In the specific case of the $S^{2} X S^{1}$ background under consideration everything becomes much simpler:

${ }_{H^{m}}=\theta^{\alpha++}{ }_{\theta} \beta^{--} e_{\alpha} \beta^{m}, \quad E^{-1}=e^{-1}+\theta^{\alpha++}{ }_{\theta} \beta--\omega_{\alpha} \gamma_{\gamma \beta}+\left(\theta^{++}\right)^{2}\left(\theta^{--}\right)^{2} R$.

Given all this, it is a matter of a relatively simple computation to find the component form of the $\mathrm{N}=2$ singleton action (note that the fields $\phi^{+}, \psi_{\alpha}{ }^{-}$have non-zero Weyl weights (see (3.12)), which can always be adjusted using dete ${ }^{m}$ ). In conclusion we stress that the $N=2$ singletons can self-interact in much the same way as those of $\mathrm{N}=1$ (in particular, upon elimination of the auxiliary fields one obtains the scalar term $\left(\phi^{+} \phi^{-}\right)^{3}$ or the spinor term $\left(\phi^{+}\right)^{2}\left(\psi^{\alpha-} \psi_{\alpha}^{-}\right)+$c.c. $)$. 
The next case we consider is $\mathrm{N}=4 \quad(\mathrm{~N}=3$ is rather similar). The internal symmetry group now is SO(4) SU(2)XSU(2), so there are spinors corresponding to each copy of $\mathrm{SU}(2)$. We shall denote them by $i, j, \ldots$ and $a, b, \ldots$ Then the Grassmann coordinate $\theta$, which is an so(4) vector, will carry an index of each type:

$$
\left\{x^{m}, \theta_{\alpha}^{i a}\right\}, \quad \overline{\theta_{\alpha}^{i a}}=C_{\alpha \beta} \varepsilon_{i j} \varepsilon_{a b} \theta^{\beta j b} .
$$

This superspace is too big and the superfields in it are highly reducible. One finds oneself in a situation very similar to that in four-dimensional $\mathrm{N}=2$ supersymmetry. The trick used there was the introduction of harmonic variables [15], allowing one to reduce the SU(2) symmetry to $U(1)$. Here we do the same with the SU(2) group acting on the indices $a, b, \ldots$ In other words, we introduce harmonic variables

$u_{a^{ \pm}}^{ \pm}, \quad u^{+a_{u^{-}}}{ }_{a}=1, \quad u^{-}{ }_{a}=\overline{u^{+a}}$

transforming under the second su(2) group (index a) and under a U(1) group (index + or - ). With their help we can split $\theta_{\alpha}$ ia into

$\theta_{\alpha}{ }^{i \pm}=\theta_{\alpha}^{i a_{a_{a}}}$

and then define a smaller superspace: 
$\left\{x_{A}^{m}, \theta_{\alpha}^{i+}, u_{a}^{ \pm}\right\}, \quad x_{A}^{m}=x^{m}+i \theta^{i a} \gamma^{m} \theta_{i} b_{u^{+}}\left(a^{-}{ }^{-} b\right)$

which is closed under the full $\mathrm{N}=4$ supersymmetry group:

$\delta \mathrm{x}_{A}{ }^{m}=2 i \varepsilon^{i-} \gamma^{m} \theta_{i}{ }^{+}, \quad \delta \theta_{\alpha^{i+}}=\varepsilon_{\alpha^{i+}}, \quad \delta u_{a}^{ \pm}=0$.

The superfields defined in (4.4) (we call them analytic since they do not depend on $\theta^{-}=\overrightarrow{\theta^{+}}$, are much simpler. For instance, a scalar superfield carrying $U(1)$ charge +1 has the following decomposition:

$\Phi^{+}\left(\mathrm{x}_{\mathrm{A}}^{\mathrm{m}}, \theta_{\alpha}^{i+}, \mathrm{u}_{\mathrm{a}}^{ \pm}\right)=$

$=\phi^{+}\left(x_{A}, u\right)+\theta^{\alpha i+} \psi_{\alpha i}\left(x_{A}, u\right)+\left(\theta^{+}\right)^{2} \alpha \beta^{i j_{A}} \alpha \beta_{i j}(-)\left(x_{A}, u\right)+$

$+\left(\theta^{+},{ }^{3 \alpha i} \chi_{\alpha i}(-2)\left(x_{A}, u\right)+\left(\theta^{+},{ }^{4}{ }_{B}(-3)\left(x_{A}, u\right)\right.\right.$.

Note that all the fields in (4.6) depend on the new variables. In other words, they have infinite expansions, e.g.,

$\phi^{+}\left(x_{A}, u\right)=\phi^{a}\left(x_{A}\right) u_{a}^{+}+\phi^{(a b c)}\left(x_{A}\right) u_{a}{ }^{+} u_{b^{+}}{ }^{+} c_{C}^{-}+\ldots$,

$\psi_{\alpha i}\left(x_{A}, u\right)=\psi_{\alpha i}\left(x_{A}\right)+\psi_{\alpha i}(a b)\left(x_{A}\right) u_{a}{ }^{+} u_{b}{ }^{-}+\ldots$.

The first terms in the expansions of $\phi^{+}$and $\psi$ are just the components of an $\mathrm{N}=4$ singleton multiplet (both are $\mathrm{SO}(4)$ spinors). All the remaining fields in (4.7) and in (4.6) must become auxiliary. The phenomenon of having an infinite set of fields off shell in a theory involving only a small number of 
fields on shell has already occurred in four-dimensional $\mathrm{N}=2$ supersymmetry, in particular in the description of the scalar multiplet (hypermultiplet) [15]. By analogy we can try the following free off-shell action for $\Phi^{+}$and its conjugate $\widetilde{\Phi}^{+}$(the conjugation $\sim$ involving the harmonic variables has been defined in [15]):

$s=\int \mathrm{d}^{3} \mathrm{x}_{\mathrm{A}} \mathrm{d}^{4} \boldsymbol{\theta}^{+} \mathrm{du} \widetilde{\Phi}^{+} \mathrm{D}^{++} \Phi^{+}$

where $\mathrm{D}^{++}$is the derivative with respect to the harmonic variables (properly supersymmetrized):

$\mathrm{D}^{++}=\mathrm{u}^{+a} \partial / \partial_{\mathrm{u}}^{-\mathrm{a}}+\mathrm{i} \boldsymbol{\theta}^{\mathrm{i}+} \boldsymbol{\gamma}^{\mathrm{m}} \boldsymbol{\theta}_{\mathrm{i}}+\partial / \partial \mathrm{X} \mathrm{m}$

Indeed, varying $\Phi^{+}$in $(4.8)$ one gets the equation of motion

$\mathrm{D}^{++} \boldsymbol{\Phi}^{+}=0$

In terms of component fields it decomposes into a number of equations. Thus, at the levels $(\theta)^{0}$ and $(\theta)^{1}$ one finds:

$$
\begin{aligned}
& \partial^{++} \phi^{+}=u^{+} \partial / \partial u^{-} \phi^{+}=0 \rightarrow \phi^{+}=\phi^{a}\left(x_{A}\right) u_{a}{ }^{+} ; \\
& \partial^{++} \psi_{\alpha i}=0 \rightarrow \psi_{\alpha i}=\psi_{\alpha i}\left(x_{A}\right) .
\end{aligned}
$$

Next, the level $(\boldsymbol{\theta})^{3}$ equation is

$$
\partial^{++} \chi_{\alpha i}{ }^{(-2)}+i \partial_{\alpha} \beta_{\Psi_{\beta i}}=0
$$


which, together with (4.12), gives

$\chi_{\alpha i}(-2)=0, \quad i \partial_{\alpha} \beta_{\psi_{i}\left(x_{A}\right)=0}$

The $\left(\theta^{+}\right)^{2}$ equation relates $A$ to $\phi^{\mathrm{a}}\left(\mathrm{x}_{\mathrm{A}}\right)$ :

$\partial^{++}{ }_{A_{i j}} \alpha \beta(-)+i \partial \alpha \beta_{\phi^{+}} \varepsilon_{i j}=0 \rightarrow A_{i j} \alpha \beta(-)=\partial \alpha \beta_{\phi^{a}}\left(x_{A}\right) u_{a}{ }^{-} \varepsilon_{i j}$,

and the $(\theta)^{4}$ one produces

$\mathrm{B}^{(-3)}=0, \quad \square \phi^{\mathrm{a}}\left(\mathrm{x}_{\mathrm{A}}\right)=0$.

The net result is that on shell all the fields present in (4.6) vanish or are expressed in terms of the physical fields $\phi^{a}, \psi_{\alpha i}$, which in turn satisfy their standard equations of motion (4.15), (4.13).

The problem of arranging self-interactions for $\boldsymbol{\Phi}^{+}$is not as simple as in the cases $N=1$ and $N=2$. Now we must make sure that the integrand in a self-interaction term has exactly the opposite charge to that of $\mathrm{d}^{4} \boldsymbol{\theta}^{+}$, otherwise the harmonic integral will vanish (see [15]). An obvious candidate is

$S_{\text {int }}=\lambda \int d^{3} x_{A} d^{4} \theta^{+} d u\left(\Phi^{+}\right)^{4}$

However, this time the dimensions do not match (now we have twice as many $\theta^{\prime} s$ as in the $N=2$ case), $[\lambda]=-1$ and the term (4.16) is not conformally invariant. Nevertheless, it is still possible to have self-interactions, provided two conditions 
hold. First, we give up the so(4) invariance of the interaction (but not of the kinetic term) and form the linear combination $\widetilde{\Phi}^{+} u_{1}--\Phi^{+} u_{2}-$ (explicit harmonic dependence). Second, we assign a non-vanishing vacuum expectation value to one of the scalars $\phi^{a}(x)$, so that we can divide by the above combination. Then the following interaction term

$$
\left(\widetilde{\Phi}^{+}\right)^{2}\left(\Phi^{+}\right)^{2}\left(\widetilde{\Phi}^{+} u_{1}--\Phi^{+} u_{2}^{-}\right)^{-2}
$$

has both the right charge +4 and dimension 1 . We stress that the resulting self-interaction for the physical fields $\phi$ and $\psi$ (upon elimination of the infinitely many auxiliary fields) is highly non-polynomial and would have been practically impossible to guess had we started from the on-shell formulation of the theory.

Introducing conformal supergravity is now even easier than in the $\mathrm{N}=2$ case. Following [16], we consider the gauge group

$$
\begin{aligned}
& \delta x_{A}^{m}=\lambda^{m}\left(x_{A}, \theta^{+}, u\right), \quad \delta \theta_{\alpha^{i+}}=\lambda_{\alpha^{i+}}\left(x_{A}, \theta^{+}, u\right), \\
& \delta u_{a}^{+}=\lambda^{++}\left(x_{A}, \theta^{+}, u\right), \quad \delta u_{a}^{-}=0 .
\end{aligned}
$$

As a consequence the derivative $\mathrm{D}^{++}$(4.9) should become fully covariant:

$$
\nabla^{++}=u^{+} \partial / \partial u^{-}+H^{(+4)} u^{-} \partial / \partial u^{+}+H^{++m} \partial / \partial x^{m}+H^{++\alpha i+} \partial \partial \theta^{\alpha i+}
$$


where the vielbeins $\mathrm{H}^{(+4)}, \mathrm{H}^{++m}, \mathrm{H}^{++\alpha i+}$ are arbitrary analytic superfields (functions of $x, \theta^{+}, u$ ). After assigning to $\Phi^{+}$the transformation law

$\Phi^{+\prime}=\omega_{A}^{-1 / 2} \Phi^{+}, \quad\left(d^{3} x_{A} d^{4} \theta^{+} d u\right)^{\prime}=\omega_{A}\left(d^{3} x_{A} d^{4} \theta^{+} d u\right)$,

one simply writes down

$S=\int d^{3} x_{A} d^{4} \theta^{+} d u 1 / 2\left(\widetilde{\Phi}^{+} \nabla^{++} \Phi^{+}-\Phi^{+} \nabla^{++} \widetilde{\Phi}^{+}\right)+S_{\text {int }} \cdot$

In our special case the vielbeins take the simple form

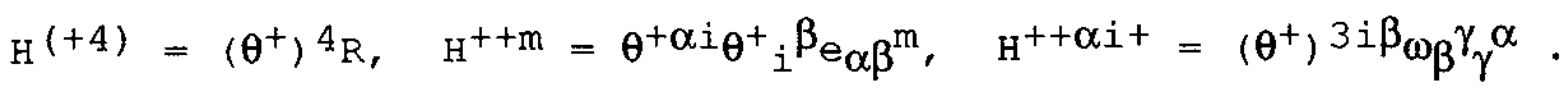

This step completes the construction of the $N=4$ singleton action.

In the case $\mathrm{N}>4$ the situation is not yet clear (remember that there are no four-dimensional analogues of such theories). A possible way of treating the $N=6$ case is to reduce the group $S O(6) \sim S U(4)$ to its subgroup $S U(3) X U(1)$ by introducing suitable harmonic variables. This option is still under investigation.

In conclusion we can say that there is sufficient evidence that the symmetry principles of the extended supersymmetric singleton theories are not strong enough to prohibit all interactions. 
Acknowledgements. I am especially grateful to M. Duff, who suggested examining the problem and provided me with a lot of information. I also benefited from discussions with C. Fronsdal and E. Sezgin. Part of this work has been done at ICTP, Trieste, and I thank Professor Abdus Salam and ICTP for their hospitality.

\section{References}

1. P. Dirac, J. Math. Phys. 4 (1963) 901

2. C. Fronsdal, Lett. Math. Phys. 1 (1976) 165

3. E. Angelopoulos, M. Flato, C. Fronsdal and D. Sternheimer, Phys. Rev. D23 (1981) 1278

4. C. Fronsdal, Phys. Rev. D26 (1982) 1988

5. M. Flato and C. Fronsdal, in "Essays on supersymmetry" (C. Fronsdal, ed.), Reidel (1986) 123

6. C. Fronsdal, preprints UCLA/88/TEP/5 and 6 (1988)

7. E. Bergshoeff, M. Duff, C. Pope and E. Sezgin, Phys. Lett. B199 (1987) 69

8. M. Blencowe and M. Duff, Phys. Lett. B203 (1988) 229

9. E. Sezgin, Y. Tanii and H. Nicolai, Trieste preprint IC/88 (1988)

10. E. Ivanov and A. Sorin, J. Phys. A 13 (1980) 1159

11. J. Gates, M. Grisaru, M. Rocek and W. Siegel, Superspace, Benjamin-Cummings, Reading (1983)

12. S. Ferrara, B. Zumino and J. Wess, Phys. Lett. B51 (1974) 239 
13. V. Ogievetsky and E. Sokatchev, Phys. Lett. B71 (1978) 222, Yad. Phys. 28 (1980) 1631, Yad. Phys. 31 (1980) 264

14. B. Zupnik, Yad. Phys. 36 (1982) 779

15. A. Galperin, E. Ivanov, S. Kalitzin, V. Ogievetsky and E. Sokatchev, Class. Quantum Grav. 1 (1984) 469

16. A. Galperin, E. Ivanov, V. Ogievetsky and E. Sokatchev, Class. Quantum Grav. 4 (1987) 1255 